\title{
Flower Image Retrieval Using Color and Shape Features and Multiple Distance Functions
}

\author{
${ }^{1}$ Khadiza Sultana Happy, ${ }^{2}$ Zannatul Azme, ${ }^{3, *}$ Mohammad Farhad Bulbul \\ ${ }^{1,2,3}$ Department of Mathematics, Jessore University of Science and Technology; \\ Khadijasultana007@gmail.com; zannatulazme1510@gmail.com; farhad@just.edu.bd
}

\begin{abstract}
It is noticeable that when flower images are observed, flower's color signifies people and often people ignore small and isolated sectors of the image. So for retrieving flower images color feature of the images is very important. Different flowers have also different shapes. So for differing flower images from another flower images shape feature is also a significant feature. In this paper, we proposed a method of retrieving flower images using their color and shape feature. We used HSV color histogram, color moments and color auto correlogram descriptors for describing color feature and HOG descriptor for describing shape feature. We calculate the similarities between the query image and the database images by employing a set of distance functions. We evaluated our result for our own dataset of 15 categories flower images.
\end{abstract}

Keywords: Flower image retrieval; HSV histogram; Color moments; HOG feature; L2 distance function.

\section{Introduction}

In ancient time people used the walls to draw some pictures to tell some information about their life. At the beginning of the twentieth century, an ever-growing number of digital images are being gathered with the introduction of digital photography. Nowadays, images play a vital role in various fields such as medical, education, and entertainment. By using computers, various techniques help us for capturing, processing, storage, and transmitting the images. Using World Wide Web users are facilitated to access data from anywhere and can utilize the images in any fields [1]. Although for this reason, the quantity of data becomes larger and larger, it will be unusable unless there are active methods to access them.

Images collected from several fields are stored in image databases. And the images for each field have certain common features. In many fields such as medical, engineering, educational, sports, criminal etc. images are used. For example, X-ray images are used in medical fields for diagnoses and research purpose. For finding the suspicious people face recognition is used in the criminal field. If we want to search an image by text or image description, the result will not accurate and it will time consuming. So it is not effectual to use text or image descriptors to search for an image in a huge image database. A new method called content-based image retrieval is used to retrieve images from the database to overwhelm this problem [2].

Content-based image retrieval (CBIR) is a process to retrieve images based on their visual features like color, texture, and shape [3]. The conception of using content-based image retrieval was first used by Kato to describe his experiments for retrieving images from a database using color and shape features. 
Nowadays CBIR methods have become a dependable tool for many image database applications. There are several benefits of CBIR techniques.

Flower image retrieval method is a type of content-based image retrieval method and it is very effectual for computer-aided flower species recognition. With the availability of digital photo devices, the collection of flower images can be enormous in size, containing hundreds, thousands or even millions of flower images in a database. There are many difficulties to retrieve flower images for being its complex backgrounds, for variants of brightness and intensity under diverse natural illuminations, shadow effects of the neighboring, different camera viewpoints, different dimensions, and different resolution, etc. As a result, the retrieval of flower images is a challenging problem in content-based image retrieval.

Many researchers have been researched on flower image retrieval system. In 2008, Maria-Elena Nilsback and Andrew Zisserman [3] make known to a flower dataset with 103 class. They compute four different features namely the local shape/texture, the shape of the boundary, the overall spatial distribution of petals, and the color of the flowers. They merged the features using a multiple kernel framework with a classifier called SVM. The weights for each class were learnt using the technique of Varma and Ray [4], which has succeeded state of the art performance on another huge dataset, such as Caltech 101/256. Their dataset had a comparable contest in the number of classes. Their results displayed that learning the optimum kernel combination of multiple features vastly improves the performance, from $55.1 \%$ for the best single feature to $72.8 \%$ for the combination of all features.

A few years later, Lin Li, Yu Qiao [5] presented a new idea for flower image retrieval. They created by using of category attributes to construct middle-level image representation. Image retrieval has been utilized by low-level features. They utilized category attributes, i.e. daisy, buttercup or iris to construct a semantic representation of flower images. They trained a linear SVM based on low-level visual features, containing the appearance of color, texture and shape for each category attribute. These classifiers are regarded as attribute features. So that, they construct a new mid-level visual presentation - category attribute - for the query and images in the dataset. They were evaluated on 17 category flower dataset. Finally, they took the result of captures flower category attribute that was more effective and had lower dimension. So they did not need to rely on costly and inefficiently similarity measurement between low-level feature vectors.

After that, Y H Sharath Kumara*, D S Gurub [6] propose a model based on a query sketch for representation and indexing of flower images for the purpose of retrieving flowers of interest. They used a subset of flower sketches having 20 users and for each user 5 flower sketches, totally about 100 sketches. Their thought was an effective way too rapid up the retrieval procedure is to design an indexing scheme initially for retrieval, which results in the reduction of the number of flowers considered for searching by the retrieval algorithm. They represented each flower by shape descriptors of Scale Invariant Feature Transform (SIFT), Histogram of Gradients (HOG) and Edge Orientation Histograms (EOH). Their Experimentations were conducted on Category-127dataset and Flower Sketch dataset (their own flower data set of 127 classes of flowers, consists of 13169 flower images) to assess the advantage of using indexing technology. Their experimentation ensures that the combination of all the features descriptors achieves a good accuracy with indexing approach. 


\section{Proposed Method}

\subsection{Architectural Overview}

In this part, we will discuss about our method for retrieving flower images. Firstly, extracting and calculating the features we create feature vectors for the query image and database images. Then using some distance functions through the feature vectors we find out the similarity between the query image and the database images. Figure 1 represents an architectural overview of the retrieval system.

Figure 1: User interface of the retrieval system

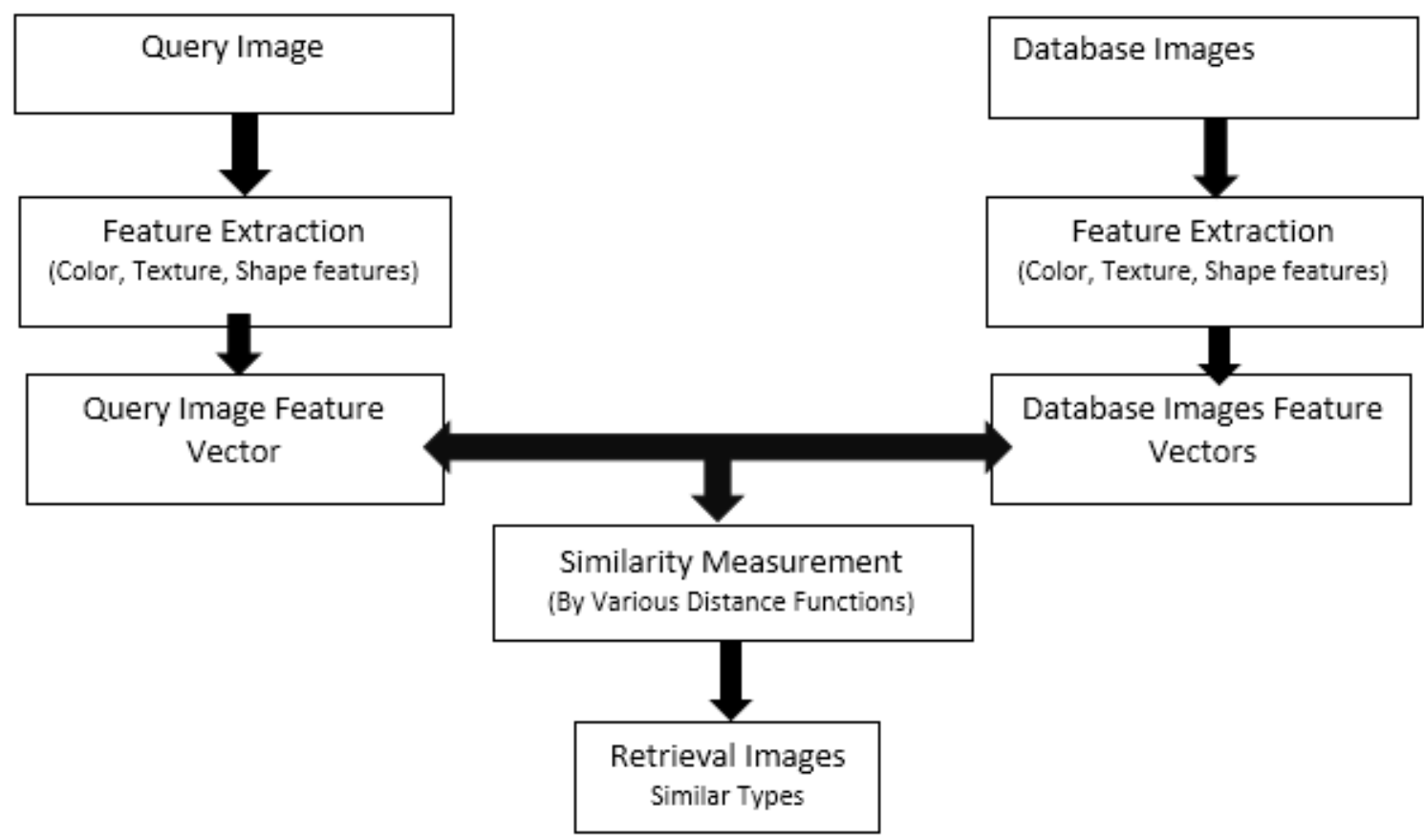

\subsection{Feature Extraction}

Using the contents of images, CBIR technique represent the image. From the value of the image pixels CBIR systems extract features (color, texture, and shape). The size of these features are smaller than the size of the images and they are stored in a database called feature database. In the feature database each image is represented by a combine representation of its contents (color, texture, shape, and spatial information) in the form of a fixed length real-valued multi-component feature vectors or signature. This is called offline feature extraction [7].

In our work we use descriptors for color features and Histogram of oriented gradients (HOG) for shape feature to describe a flower image. This section discusses those features briefly.

\subsubsection{Color Feature}

Color is the most spontaneous feature of an image and to describe colors HSV histogram, color moments and color auto correlogram are used in our method. Histogram techniques have the advantages of rapidness, lower demand of memory space. Color features are the most significant elements facilitating human to recognize images. For categorizing images, powerful information can be provide by color features and they are used for image retrieval. 
(i)HSV histogram: Anyone with a monitor has probably heard of the RGB color space (Jacci Howard bear). HSV is a variation of an RGB color space, and its components and colorimetry are comparative to the RGB color space from which it was obtained. We may have noticed HSV in the color picker of our graphics software when we deal with commercial printers. HSV are common cylindrical-coordinate representations of points in an RGB color model. The HSV color model describes in terms of hue, saturation and value (brightness). Hue corresponds right away to the opinion of hue in the color Basics section. It is the dominate color which is perceived by an observer. Saturation corresponds directly to the concepts of tint in the color . Basics section except that full saturation produces on tint while zero saturation produces white a shade of gray, or black. Value corresponds directly to the concepts of intensity in the color Basics section. The HSV color wheel is sometimes illustrated as a cone or cylinder. But it always with these three components. It is used when choosing colors for draw or ink as HSV better represents how people relate to colors than dose the RGB color space. Selecting an HSV color starts with picking one of the available hues, which is how most humans anticipate to color, and then conforming the shade and brightness value. The benefit of HSV is that each of its quality corresponds right away to the basic color thought, which makes it conceptually instinctive. RGB to HSV conversion formula [8] is shown as follows:

The $R, G, B$ values are distributed by 255 to change the range from $0 . .255$ to $0 . .1$ :

$R^{\prime}=R / 255$

$G^{\prime}=G / 255$

$B^{\prime}=B / 255$

$C \max =\max \left(R^{\prime}, G^{\prime}, B^{\prime}\right)$

$C \min =\min \left(R^{\prime}, G^{\prime}, B^{\prime}\right)$

$\Delta=C \max -C \min$

Hue calculation:

$$
H=\left\{\begin{array}{lr}
0^{\circ} & \Delta=0 \\
60^{\circ} \times\left(\frac{G^{\prime}-B^{\prime}}{\Delta} \bmod 6\right), C_{\max }=R^{\prime} \\
60^{\circ} \times\left(\frac{B^{\prime}-R^{\prime}}{\Delta}+2\right), C_{\max }=G^{\prime} \\
60^{\circ} \times\left(\frac{R^{\prime}-G^{\prime}}{\Delta}+4\right), C_{\max }=B^{\prime}
\end{array}\right.
$$

Saturation calculation:

$$
S=\left\{\begin{aligned}
0, & C_{\max }=0 \\
\frac{\Delta}{C_{\max }}, & C_{\text {max }} \neq 0
\end{aligned}\right.
$$

Value calculation:

$$
V=C_{\max }
$$

Where,

$H($ Hue $)=$ Dominant color(spectral), $S($ saturation $)=$ Amount of white, $V($ values $)=$ Brightness 
Figure 2: Example of HSV images of flower images

Original Images $\longrightarrow$ HSV Images
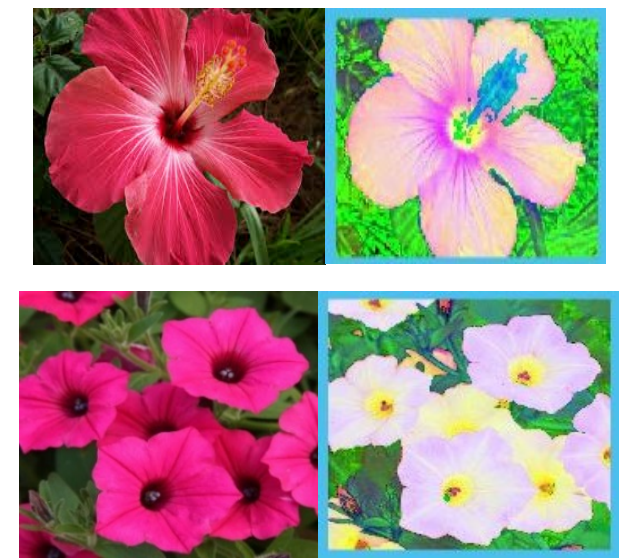

(ii) Color moments: Color moments have been successfully used in content based image retrieval system. It is used for extraction of color feature. It can be used differentiate images based on their features of color are measured by color moments. The number of color distribution in an image in the identical way are measured by it. It is measures central moments uniquely relating a probability distribution. It is mainly used for color indexing purposes as features in image retrieval applications. Probability distribution are characterized by a number of unique moments. Color moments are scaling and rotation invariant. It can be computed for any color model. Stricker and Orengo [9] use three central moments of an image's color distribution. They are Mean, Standard deviation and Skewness. A color can be described by 3 or more values. It is generally the case that only the first three color moments are used as features in color image retrieval system that is HSV hypothesis of Hue, saturation and value. Three color moments are computed per channel. An image therefore is characterized by 9 moments-3 moments for each color channels. Calculating color moments is done in the same way as computing moments of a probability distribution. Now we will identify the $\mathrm{m}$-th color direction at the $\mathrm{n}$-th image pixel as $p_{m n}$. The three color moments can then be defined as,

Mean: It can be calculated by using the following formula,

$$
E_{m}=\sum_{n=1}^{N} \frac{1}{N} p_{m n}
$$

Where,

$\mathrm{N}=$ the number of pixels in the images

$p_{m n}=$ the value of the $\mathrm{n}$-th pixel of the image at the $\mathrm{m}$-th color channel.

Mean can be assumed as the middling color value in the image.

Standard Deviation: It can be calculated by using the following formula,

$$
\sigma_{m}=\sqrt{\left(\frac{1}{N} \sum_{n=1}^{N}\left(p_{m n}-E_{m}\right)^{2}\right)}
$$

Where

$E_{m}=$ The mean value or first color moment for the m-th color channel.

The standard deviation is the square root of the adaptation of the distribution.

Skewness: It can be calculated by using the following formula, 


$$
\left.s_{m}=\sqrt[3]{\left(\frac{1}{N}\right.} \sum_{n=1}^{N}\left(p_{m n}-E_{m}\right)^{3}\right)
$$

Skewness can be perceive as a measure of the dimension of asymmetry in the distribution.

(iii) Color auto correlogram: Color is an important visual feature. We have been successfully used of color auto correlogram algorithm in color based image retrieval as a feature descriptor. It is used for extraction of color feature. Correlogram can be conserved as a table indexed by pairs of colors $(m, n)$ where $d$-th entry shows the probability of finding a pixel $n$ from pixel $m$ at distance $d$. Whereas a color auto-correlogram can be conserved as a table index of color in the same pixel at distance $d$. Hence auto-correlogram captures spatial correlation into same colors only [10].

Let [D] denote a set of $\mathrm{D}$ given distances $\mathrm{d} 1$ ? $\mathrm{dD}$. Then the correlogram of the image $\mathrm{M}$ is defined for level pair $g_{m} ; g_{n}$ at a distance $d$ is given by,

$$
\gamma_{g_{m}, g_{n}}^{d}(M)=P_{s_{p 1 \in M g_{m}, p 2 \in M}}\left[p 2 \in M_{g_{n}}\|p 1-p 2=d\|\right]
$$

This gives the probability that given any pixel p1 of level $g_{m}$, a pixel p2 at a distance $d$ in certain direction from the given pixel $\mathrm{p} 1$ is of level $g_{m}$.

Color auto correlogram captures the spatial correlation of same levels only [11]:

$$
\alpha_{g}^{d}(M)=\gamma_{g_{m} g_{n}}^{d}(M)
$$

It gives the probability that pixels $\mathrm{p} 1$ and $\mathrm{p} 2, \mathrm{~d}$ away from each other, are of the same level $g_{m}$.

\subsubsection{Shape Feature}

The physical structure of the objects, or the geometric shapes present in the image. Objects may be recognized from their outline. Shape is very powerful feature. Shape feature must present some essential properties such as identifiability, translation, rotation and scale invariance, noise resistance, occultation invariance, statistically independent, reliability etc. [12]

(i) Histogram of oriented gradients (HOG): The histogram of oriented gradients (HOG) is a feature descriptor used in computer vision. The HOG feature are widely use of image processing for the purpose of object detection. The detector can be distributed into HOG feature extraction and SVM classification. HOG feature is used for extraction of shape feature. HOG decomposes an image into small squared cells. It counts a histogram of oriented gradients in every cell. It also normalizes the result using a block-wise Patten then return a descriptor for each cell. HOG is easy to express the rough shape of the object. It is robust to variations in geometry. But HOG is not supported into rotation and scale changes [13]. HOG feature used in algorithm such as color image is converted to grayscale, luminance gradient is calculated at each pixel.

When calculate a HOG descriptor, we need to first calculate the horizontal and vertical gradients. Because we want to calculate the histogram of gradients. The horizontal and vertical gradients by filtering the kernels as the table below, then calculated the histogram of gradients,

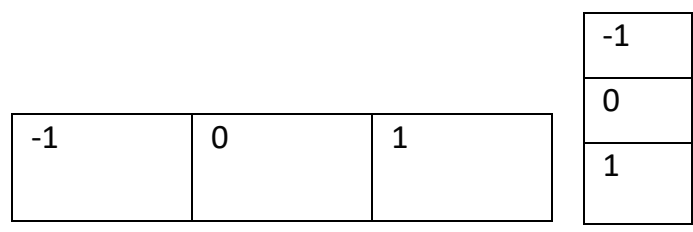


We know that, gradient basically is defined as a measure of change in an image function. Gradient of an image function is defined as mathematically given as follows,

Let, $F(X, Y)$ is the image function. Where, $\mathrm{X}$ represents the horizontal direction and $\mathrm{Y}$ represents the vertical direction.

$$
\text { Centered: } F^{\prime}=\lim _{h \rightarrow 0} \frac{f(x+h)-f(x)}{h}
$$

$$
\text { Gradient of an image } \quad \nabla F=\left[\frac{\delta F}{\delta x}, \frac{\delta F}{\delta y}\right]
$$

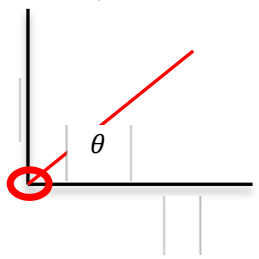

Where,

$$
F_{x}=\frac{\delta F}{\delta x}=F(x+1)-F(x),
$$

Which is denoted by differential of the function image $F$ w.r.to $X$.

$$
F_{y}=\frac{\delta F}{\delta y}=F(y+1)-F(y)
$$

Which is denoted by differential of the function image $F$ w.r.to $y$.

This is the way to measure of change in image function $F$ in both $x$ and $y$.

Now we can find the magnitude, direction and normalization of gradient using the following formula,

$$
\begin{array}{lc}
\text { Gradient magnitude } & \|\nabla F\|=\sqrt{\left(\frac{\delta F}{\delta x}\right)^{2}+\left(\frac{\delta F}{\delta y}\right)^{2}} \\
\text { Gradient direction } & \theta=\tan ^{-1}\left[\frac{\delta F}{\delta y} / \frac{\delta F}{\delta x}\right] \\
\text { Normalization } & N=\frac{\nabla F}{|\nabla F|}
\end{array}
$$

HOG create a histogram of gradient orientations for each cell. Then HOG calculated normalization and descriptor blocks. To calculate the final feature vector HOG descriptors may be used for a machine learning algorithm.

Figure 3 : HOG representaion of flower images


HOG
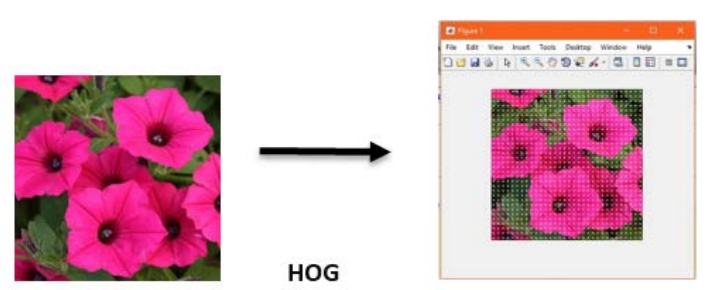


\subsection{Distance Function}

For measuring distance between two points in a vector space, the thought of distance function has been established. In the part, we discuss about six distance function namely L2, chebyshev, cosine, correlation, city block and Minkowski. These distance functions are used to measure similarities between query image feature vector and the database image feature vectors.

(i) L2 Distance Function: The L2 distance between two points' $p$ and $q$ is the length of the line segment between them.

If $p=\left(p_{1}, p_{2}, \ldots p_{m}\right)$ and $b=\left(q_{1}, q_{2}, \ldots q_{m}\right)$ are two points in Euclidian $\mathrm{n}$-space then the distance $\mathrm{d}$ between $p$ and $q$ is obtain by

$$
\begin{aligned}
& d(p, q)=\sqrt{\left(p_{1}-q_{1}\right)^{2}+\left(p_{2}-q_{2}\right)^{2}+\cdots+\left(p_{m}-q_{m}\right)^{2}} \\
& \quad=\sqrt{\sum_{j=1}^{m}\left(p_{j}-q_{j}\right)^{2}}
\end{aligned}
$$

Where $p$ and $q$ is presented as Euclidian vectors. They are starting from the origin of the space with their terminal points and ending at the two points.

$$
\begin{aligned}
& \text { Euclidean norm of } \mathrm{a} \text { is, }\|p\|=\sqrt{p \cdot p}=\sqrt{p_{1}^{2}+p_{2}^{2}+\ldots+p_{m}^{2}} \\
& \text { And } \quad \mathrm{b} \text { is, }\|q\|=\sqrt{q \cdot q}=\sqrt{{q_{1}{ }^{2}+q_{2}{ }^{2}+\ldots+q_{m}^{2}}^{2}}
\end{aligned}
$$

The $L 2$ distance between $p$ and $q$ is

$$
\begin{aligned}
\| q & -p \|=\sqrt{(q-p) \cdot(q-p)} \\
& =\sqrt{\|q\|^{2}+\|p\|^{2}-2 p \cdot q}
\end{aligned}
$$

Chebyshev Distance Function: Chebyshev distance function observes the total magnitude of the difference between coordinates of a couple of objects. [14]

$$
\text { The formula is, } \quad d_{s}=\left|x_{m k}-x_{n k}\right|
$$

Cosine Distance function: Cosine Similarity is used for finding the similarity between the query flower image feature vector and the dataset flower image feature vectors. The similarity between two nonzero vectors of an inner product space can be measure by this function. The cosine of the angle between the vectors is measured by cosine distance function. [15]

The formula is,

$$
A \cdot B=\|A\|\|B\| \cos \theta
$$

Then the similarity is measured as,

$$
\begin{aligned}
& \text { Similarity, } \cos \theta=\frac{A \cdot B}{\|A\|\|B\|} \\
& =\frac{\sum_{i=1}^{n} A_{i} B_{i}}{\sqrt{\sum_{i=1}^{n} A_{i}^{2}} \sqrt{\sum_{i=1}^{n} B_{i}^{2}}}
\end{aligned}
$$

Where, $A_{i} B_{i}$ are the components of $A B$ vectors.

Correlation distance function: Correlation measures similarity rather than dissimilarity. It is identical angular separation by centering the coordinates to its mean value.Correlation distance function 
Khadiza Sultana Happy, Zannatul Azme, Mohammad Farhad Bulbul; Flower Image Retrieval Using Color and Shape Features and Multiple Distance Functions, Advances in Image and Video Processing, Volume 6 No 6 , December (2018); pp: 8-21

computes both linear and nonlinear association between two vectors. Calculating the distance correlation between two random vectors its compare this value to the distance correlations of many shuffles of the data.

The formula is [16],

$$
d_{s}=\frac{\sum_{i=1}^{m}\left(x_{a i}-\overline{x_{a}}\right) \cdot\left(x_{b i}-\overline{x_{b}}\right)}{\left(\sum_{i=1}^{m}\left(x_{a i}-\overline{x_{a}}\right)^{2} \cdot \sum_{i=1}^{m}\left(x_{b i}-\overline{x_{b}}\right)^{2}\right)^{\frac{1}{2}}}
$$

City block distance function: The city block distance is described as if we think about two points in the $x y$-plane. The city block distance is instead calculate as the distance in $x$ plus and the distance in $y$. This is similar to the way instead of going straight through, if we move around the buildings in a city.

The City block distance between two points, $A$ and $B$, with the dimensions $\mathrm{m}$ is determined as [17]:

$$
\begin{gathered}
\text { City block distance , } D=\sum_{i=1}^{m}\left|A_{i}-B_{i}\right|, \quad D \geq 0 \\
D=0 \text {, for identical points } \\
D>0 \text {, for Showing little similarity points }
\end{gathered}
$$

Minkowski Distance Function: The Minkowski distance is a distance in a normed vector space which can be measured as a simplification of both the Euclidean distance and the city block distance.

The Minkowski distance of order $\mathrm{n}$ between two points [18]

$$
\begin{aligned}
& P=\left(p_{1}, p_{2}, \ldots, p_{m}\right) \text { and } V=\left(q_{1}, q_{2} \ldots q_{m}\right) \in \mathbb{R}^{n} \\
& D(P, Q)=\left(\sum\left|p_{i}-q_{i}\right|^{n}\right)^{\frac{1}{n}}
\end{aligned}
$$

When, $n<1$, the distance between $(0,0)$ and $(1,1)$ is $2^{\frac{1}{n}}>2$

\section{Experimental Results and Discussion}

This section firstly discusses the image dataset construction and then the experiments to evaluate the proposed method using the dataset are carried out.

\subsection{Image Dataset}

Downloading the flower images from Google we make a flower image dataset. The dataset contains 300 flower images of 15 species with 25 flower images of each specie. Thus, it is difficult to detach the flower images from one another, we actually arrange our flower dataset in this way so that this type of problems can arise and we try to introduce a process to solve those problems. Figure 4 represents a portion of our dataset of different flower images.

Figure 4: A portion of flower image dataset

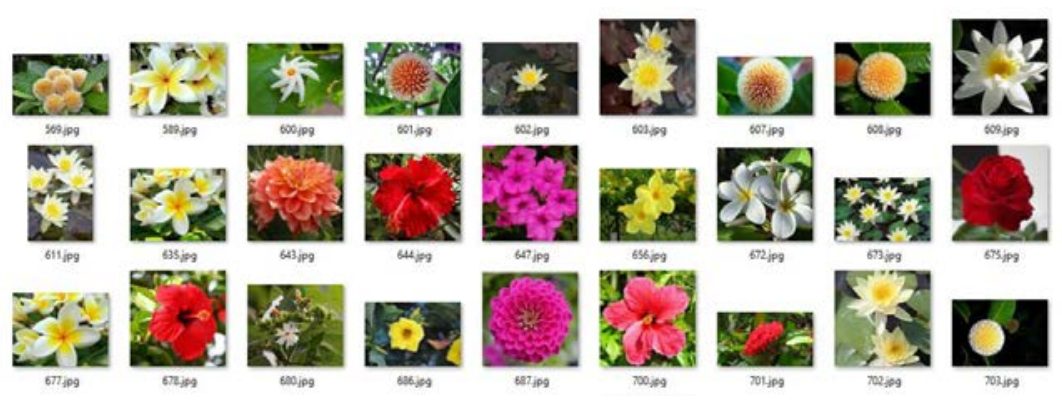




\subsection{Retrieval Results on Individual Features}

\subsubsection{Color Feature Based Image Retrieval}

For describing color feature we use three descriptor i.e. HSV histogram, color auto correlogram and color moments. We first calculate results for every descriptor individually.

For describing color feature we use three descriptor i.e. HSV histogram, color auto correlogram and color moments. We first calculate results for every descriptor individually.

(i) For HSV histogram feature we calculate accuracy rate of retrieving similar type of images and represent them at the following table.

Table 1: Retrieval accuracy using HSV histogram for color feature

\begin{tabular}{|c|c|c|c|}
\hline $\begin{array}{c}\text { Distance } \\
\text { Function }\end{array}$ & $\begin{array}{c}\text { Retrieval } \\
\text { Target }\end{array}$ & $\begin{array}{c}\text { No. of Retrieve } \\
\text { Image }\end{array}$ & $\begin{array}{c}\text { Average } \\
\text { accuracy (\%) }\end{array}$ \\
\hline L2 & $\mathbf{2 0}$ & $\mathbf{8}$ & $\mathbf{4 0}$ \\
\hline City block & $\mathbf{2 0}$ & $\mathbf{8}$ & $\mathbf{4 0}$ \\
\hline Minkowski & 20 & 5 & 20 \\
\hline Chebychev & 20 & 5 & 25 \\
\hline Cosine & 20 & 6 & 30 \\
\hline Correlation & 20 & 5 & 26 \\
\hline
\end{tabular}

The accuracy rate of retrieving similar type of images using HSV histogram for color feature is presented by a graph at the following figure.

For color auto correlogram feature we calculate the result and represent them at the Table 2.

Table 2: Retrieval accuracy using color auto correlogram for color feature

\begin{tabular}{|c|c|c|c|}
\hline $\begin{array}{c}\text { Distance } \\
\text { Function }\end{array}$ & $\begin{array}{c}\text { Retrieval } \\
\text { Target }\end{array}$ & $\begin{array}{c}\text { No. of Retrieve } \\
\text { Image }\end{array}$ & $\begin{array}{c}\text { Average } \\
\text { accuracy (\%) }\end{array}$ \\
\hline L2 & 20 & 3 & 15 \\
\hline City block & 20 & 3 & 15 \\
\hline Minkowski & 20 & 2 & 10 \\
\hline Chebychev & $\mathbf{2 0}$ & $\mathbf{4}$ & $\mathbf{2 0}$ \\
\hline Cosine & 20 & 2 & 10 \\
\hline Correlation & 20 & 2 & 10 \\
\hline
\end{tabular}

For color moments feature we calculate the result and represent them at table given bellow.

Table 3: Retrieval accuracy using color moments for color feature

\begin{tabular}{|c|c|c|c|}
\hline Distance Function & $\begin{array}{c}\text { Retrieval } \\
\text { Target }\end{array}$ & $\begin{array}{c}\text { No. of } \\
\text { Retrieve } \\
\text { Images }\end{array}$ & $\begin{array}{c}\text { Average } \\
\text { accuracy (\%) }\end{array}$ \\
\hline L2 & 20 & 5 & 25 \\
\hline City block & 20 & 5 & 25 \\
\hline Minkowski & 20 & 6 & 30 \\
\hline Chebychev & 20 & 5 & 25 \\
\hline Cosine & 20 & 6 & 30 \\
\hline Correlation & $\mathbf{2 0}$ & $\mathbf{8}$ & $\mathbf{4 0}$ \\
\hline
\end{tabular}


Khadiza Sultana Happy, Zannatul Azme, Mohammad Farhad Bulbul; Flower Image Retrieval Using Color and Shape Features and Multiple Distance Functions, Advances in Image and Video Processing, Volume 6 No 6 , December (2018); pp: 8-21

The accurate rate of retrieving similar type of images using HSV histogram, color moments and auto correlogram for color feature is presented by a graph at Figure 5.

Figure 5: Comparison of retrieval rates for HSV histogram, color auto correlogram and color moments and some distance function

\section{Acurate retrieval rate on HSV Histogram, Auto correlogram and Color moments For Color Feature}

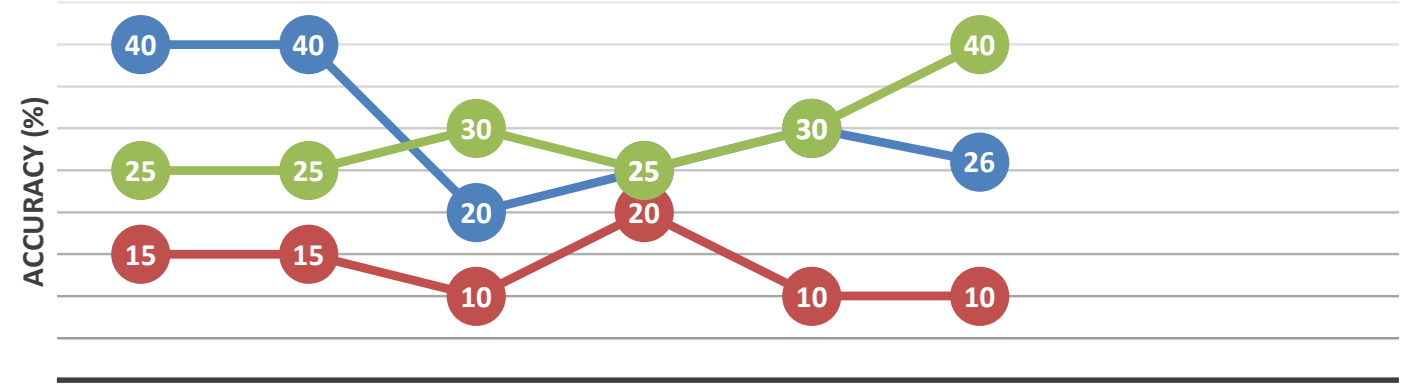

L2

CITYBLOCK MINKOWSKI CHEBYCHEV COSINE CORRELATION

DISTANCE FUNCTION

$\longrightarrow$ HSV Histrogram $\longrightarrow$ auto correlogram $\longrightarrow$ color moments

Observing the above graph we can agree that the HSV histogram is better than auto correlogram and color moments. It is observable that, HSV histogram giving $40 \%$ accurate result for the distance function L2 and city block. Color moments is also a good feature and its gives $40 \%$ accuracy for correlation distance function. So it is noticeable that for the L2 and city block distance function HSV histogram feature retrieve $40 \%$ accurate images where color moments feature give the same result for the correlation distance function. The results for auto correlogram feature are poor. Its maximum accuracy is only $20 \%$ for chebychev distance function.

(iv) We retrieve flower images based on only their color feature. We consider the color feature as a set of three features which are HSV histogram, color auto correlogram and color Moments of the query image. Then we evaluate the results for different distance functions and present in Table 4.

Table 4: Retrieval accuracy using color feature (HSV histogram, color moments, auto correlogram)

\begin{tabular}{|c|c|c|c|}
\hline Distance Function & $\begin{array}{c}\text { Retrieval } \\
\text { Target }\end{array}$ & $\begin{array}{c}\text { No. of Retrieve } \\
\text { Images }\end{array}$ & $\begin{array}{c}\text { Average } \\
\text { accuracy (\%) }\end{array}$ \\
\hline L2 & 20 & 5 & 25 \\
\hline City block & 20 & 4 & 20 \\
\hline Minkowski & 20 & 9 & 45 \\
\hline Chebychev & 20 & 10 & 50 \\
\hline Cosine & 20 & 10 & 50 \\
\hline Correlation & 20 & 10 & 50 \\
\hline
\end{tabular}

We represent the table on color feature (HSV histogram, color moments, auto correlogram) at the Figure 6. 
Figure 6: Comparison retrieval rates for HSV histogram, color moments, auto correlogram and different distance function.

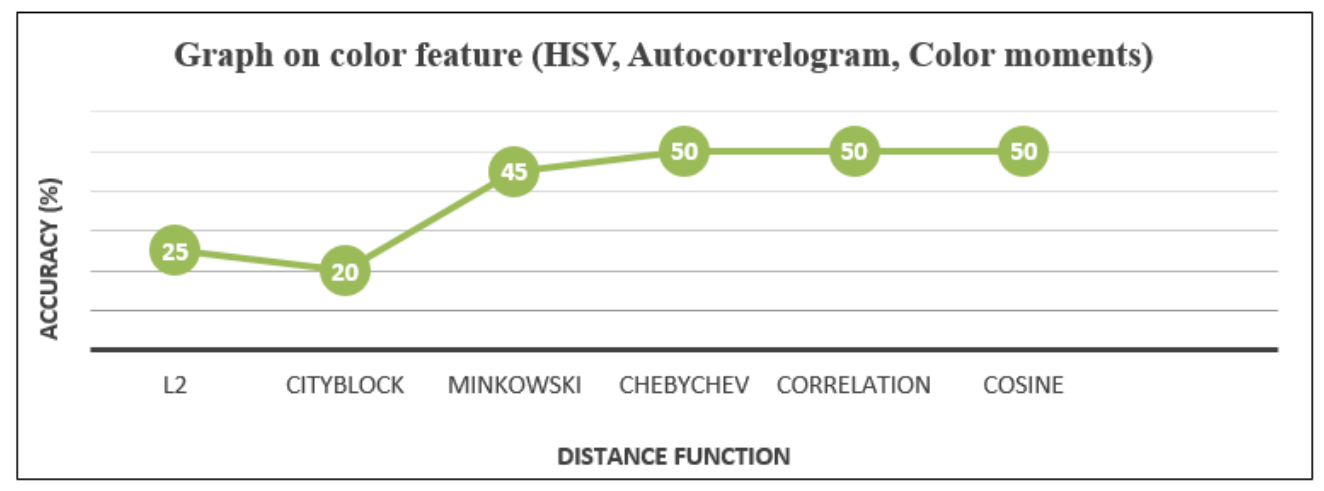

\subsubsection{Shape Feature Based Image Retrieval}

We apply our method to retrieve flower images based on only their shape feature described by HOG descriptor. Then we evaluate the results for different distance functions and construct the table 5 .

Table 5: Retrieval accuracy using shape feature

\begin{tabular}{|c|c|c|c|}
\hline Distance Function & Retrieval Target & $\begin{array}{c}\text { No. of Retrieve } \\
\text { Image }\end{array}$ & $\begin{array}{c}\text { Average accuracy } \\
\text { (\%) }\end{array}$ \\
\hline L2 & 20 & 3 & 15 \\
\hline City block & 20 & 2 & 10 \\
\hline Minkowski & $\mathbf{2 0}$ & $\mathbf{4}$ & $\mathbf{2 0}$ \\
\hline Chebychev & 20 & 3 & 15 \\
\hline Cosine & 20 & 3 & 15 \\
\hline Correlation & 20 & 5 & 25 \\
\hline
\end{tabular}

\subsubsection{Combine Feature Based Image Retrieval:}

We try to retrieve flower images based on their color and shape features. Again we compare the results for different distance functions and construct in the table 6 .

Table 6: Retrieval accuracy for combining color and shape feature

\begin{tabular}{|c|c|c|c|}
\hline $\begin{array}{c}\text { Distance } \\
\text { Function }\end{array}$ & Retrieval Target & $\begin{array}{c}\text { No. of Retrieve } \\
\text { Images }\end{array}$ & $\begin{array}{c}\text { Average } \\
\text { accuracy (\%) }\end{array}$ \\
\hline L2 & 20 & 5 & 25 \\
\hline City block & 20 & 3 & 15 \\
\hline Minkowski & 20 & 7 & 35 \\
\hline Chebychev & 20 & 6 & 30 \\
\hline Cosine & 20 & $\mathbf{7}$ & 35 \\
\hline Correlation & 20 & 6 & 30 \\
\hline
\end{tabular}

Figure 7 is representing the accuracy rate of retrieving similar images using color and shape Features. 
Khadiza Sultana Happy, Zannatul Azme, Mohammad Farhad Bulbul; Flower Image Retrieval Using Color and Shape Features and Multiple Distance Functions, Advances in Image and Video Processing, Volume 6 No 6 , December (2018); pp: 8-21

Figure 7: Evaluation of retrieval rates for using color (HSV, color auto correlogram, color moments), shape (HOG) features and several distance function

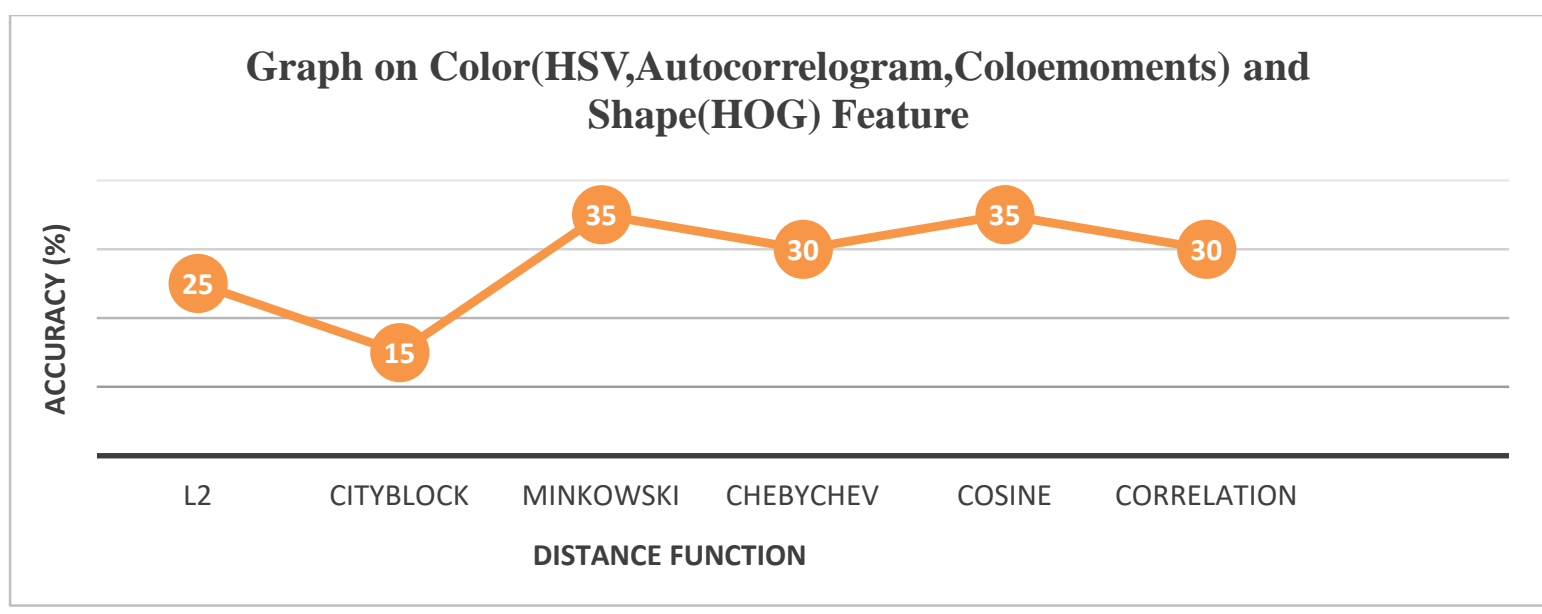

From the graph it is noticeable that the combination of color and Shape feature gives a good result for the correlation distance function.

\section{Conclusion}

In this paper, we have calculated separately the retrieval accuracy for retrieving similar type of flower images for three descriptors such as HSV histogram, color moments and auto correlogram. We observe that the result for HSV histogram is better than the other color features. We have also calculated retrieval outcomes for combining HSV histogram, color moments and auto correlogram. The retrieval results achieve an improvement for the combination of those features and better than the situation when the features are used alone. Furthermore, there is a difference in results for the different distance functions with the features. Some distance functions are giving higher results for some features only. The Minkowski and the correlation function is good for shape feature. The Minkowski and the cosine function is good when combine the color and shape features.

\section{REFERENCES}

[1] Eakins, John P., and Margaret E. Graham. "Content based image retrieval: A report to the JISC technology applications programme." (1999).

[2] Afifi, Ahmed J., and Wesam M. Ashour. "Image retrieval based on content using color feature." International Scholarly Research Notices 2012 (2012).

[3] Nilsback, Maria-Elena, and Andrew Zisserman. "Automated flower classification over a large number of classes." Computer Vision, Graphics \& Image Processing, 2008. ICVGIP'08. Sixth Indian Conference on. IEEE, 2008.

[4] Varma, Manik, and Debajyoti Ray. "Learning the discriminative power-invariance trade-off." Computer Vision, 2007. ICCV 2007. IEEE 11th International Conference on. IEEE, 2007.

[5] Li, Lin, and Yu Qiao. "Flower image retrieval with category attributes." Information Science and Technology (ICIST), 2014 4th IEEE International Conference on. IEEE, 2014.

[6] Kumara, YH Sharath, and D. S. Gurub. "Retrieval of flower based on sketches." Procedia Computer Science 46 (2015): 1577-84. 
[7] Long, Fuhui, Hongjiang Zhang, and David Dagan Feng. "Fundamentals of content-based image retrieval." Multimedia Information Retrieval and Management. Springer, Berlin, Heidelberg, 2003. 126.

[8] https://stackoverflow.com/questions/4063965/how-can-i-convert-an-rgb-image-to-grayscale-butkeep-one-color.

[9] Stricker, Markus Andreas, and Markus Orengo. "Similarity of color images." Storage and Retrieval for Image and Video Databases III. Vol. 2420. International Society for Optics and Photonics, 1995.

[10] Hazra, Dipankar. "Retrieval of color image using color correlogram and wavelet filters." Proc. of International Conference on Advances in Computer Engineering. 2011.

[11] Singla, Amit, and Meenakshi Garg. "Cbir approach based on combined hsv, auto correlogram, color moments and gabor wavelet." International Journal of Engineering and Computer Science 3.10 (2014).

[12] Yang, Mingqiang, Kidiyo Kpalma, and Joseph Ronsin. "A survey of shape feature extraction techniques." (2008): 43-90.

[13] https://en.wikipedia.org/wiki/Histogram_of_oriented_gradients

[14] https://lyfat.wordpress.com/2012/05/22/euclidean-vs-chebyshev-vs-manhattan-distance/

[15] https://en.wikipedia.org/wiki/Cosine_similarity

[16] http://people.revoledu.com/kardi/tutorial/Similarity/Correlation.html

[17] https://docs.tibco.com/pub/spotfire/7.0.0/doc/html/hc/hc_city_block_distance.htm

[18] https://en.wikipedia.org/wiki/Minkowski_distance 